\title{
Study of the effect of solvent on acetylate cashew gum-based nanoparticles properties and antimicrobial activity
}

\author{
Karolina Carneiro Morais Macedo ${ }^{1}$, Luíse Lopes Chaves ${ }^{1}$, Alexandre Couto Carneiro Vieira ${ }^{2}$ \\ Daniela Nadvorny ${ }^{2}$, Regina Célia Monteiro de Paula ${ }^{3}$, Durcilene Alves Silva ${ }^{1}$, \\ Francisco Veiga ${ }^{4}$, António José Ribeiro ${ }^{4}$, Edson Cavalcanti Silva-Filho ${ }^{1}$
}

\author{
${ }^{1}$ Laboratório interdisciplinar de Materiais Avançados - LIMAV, Universidade Federal do Piauí, Teresina, \\ Piauí, Brasil. \\ ${ }^{2}$ Núcleo de Contr. de Qualidade de Medicamentos e Correlatos - NCQMC, Universidade Federal de Pernam- \\ buco, Recife, Pernambuco, Brasil. \\ ${ }^{3}$ Laboratório de Polímeros, Universidade Federal do Ceará, Fortaleza, Ceará, Brasil. \\ ${ }^{4}$ Laboratório de Tecnologia Farmacêutica, Faculdade de Farmácia, Universidade de Coimbra, Coimbra, Por- \\ tugal. \\ e-mail:karolinacarneirom@gmail.com, luiselopes@gmail.com, alexandrecoutovieira@hotmail.com,nady@ufpe.br, \\ rpaula@dqoi.ufc.br, durcileneas@gmail.com, veiga@ci.uc.pt, aribeiro@ff.uc.pt, edsonfilho@ufpi.edu.br.
}

\section{ABSTRACT}

The aim of this work was to investigate the influence of acetone and dimethyl sulfoxide (DMSO) on acetylated cashew gun (ACG) nanoparticles parameters and to assess their antimicrobial activity. C1 nanoparticles presented smaller size $(81.3 \mathrm{~nm})$ than compared to $\mathrm{C} 2(115.7 \mathrm{~nm})$. Although, acetone formed particles with lower PDI value. Both $\mathrm{C} 1$ and $\mathrm{C} 2$ presented negative zeta potential (-39.8 and -31.7 respectively). Particle tracking analysis showed that $\mathrm{C} 1$ was slightly more concentrated than $\mathrm{C} 2$. No statistical differences (p < 0.05 ) between the same samples after 5 months were found. Both samples did not exert inhibitory effect on $E$. coli, on the contrary $S$. aureus, in which both C1 $(36.9 \%)$ and C2 $(19.9 \%)$ provided important inhibitory effect. $\mathrm{C} 1$ presented a more pronounced antifungal effect $(81.2 \%)$, compared to $\mathrm{C} 2(18.8 \%)$, indicating a promising platform to be used in several biomedical application.

Keywords: polysaccharide, nanoparticles, biopolymers, modification, biological applications of polymers.

\section{INTRODUTION}

Nanoscale biopolymer particles have been attracted much interest in research field particularly for design and fabrication of new devices for drug delivery systems and applications[1]. Moreover, their singular advantageous features as high surface area and very small size has been gain attention in the field of biomedical application as they are able to overpass barriers, which is of high interest in response to the growing threat of microbial drug resistance[2]. Nanoparticles biocide effectiveness is suggested to occur due to the ability to intimate interact with microbial cell membranes/walls and key proteins/enzymes can both inhibit pathogen growth and/or promote cell death, which may represent an alternative to the antibiotics whose resistance has been developed [2,3].

Nanoparticles can be prepared from a variety of materials, although polymeric nanoparticles obtained from natural polymers are playing an important role in drug delivery systems[4,5]. Among natural polymers used for this purpose, polysaccharides have superior features as high safety, stability, in addition to be biodegradable, and biocompatible, have abundant resources in nature and low cost in their processing[6].

Moreover, they have the advantage that can be chemically modified aiming to tailoring their physicochemical properties towards an improvement of drug concentration in the site of action without suffering degradation[5,7].

Cashew gum (GG) is a polysaccharide extracted from Anacardium occidentale tree, widely present in the northeastern part of Brazil [5,8]. It is reported to contain: b- D-galactose (72\%), D-glucose (14\%), arabinose $(4.6 \%)$, rhamnose $(3.2 \%)$ and glucuronic acid (4.7\%) [8]. This complex heteropolysaccharide, non-toxic and 
versatile biopolymer has been used as promising material in the field of pharmaceutical, and biomedical application[7,9-12].

Studies regarding the biological activities of CG demonstrated that it has antimicrobial activity against several microorganisms including gram-positive bacteria[11] and resistant strains[13]. Its antimicrobial activity has been attributed to the presence of anacardic acid[13]. Modifications in CG structure have been exploited to improve its technological properties[7,9,10]aiming to allow a more specific drug delivery and increase the drug incorporation efficiency into the matrix by increasing its hydrophobicity $[5,10,14]$.

Acetylation reactions is commonly used to replace polysaccharides hydroxyl sites with acetyl functional groups, resulting in several effects on the functional properties of the molecule, giving rise to hydrophobic modified polysaccharides[14].

Few studies have been reported the use of acetylated CG nanoparticles for drug delivery purposes[7,10] using the dialysis method and DMSO as solvent. Although, until now, no antimicrobial activity of modified CGbased nanoparticles has been reported.

The aim of this work was to investigate the influence of different solvent, namely acetone and dimethyl sulfoxide (DMSO), on nanoparticles parameters as well as to study antimicrobial activity of the obtained nanoparticles. The nanoparticles were characterized regarding size, polydispersity index and zeta potential. Particles tracking analysis (PTA) was conducted to evaluate the concentration of the nanoparticles in suspension, and colloidal stability was performed to investigate changes in zeta potential. Antimicrobial activity was performed using gram-negative, gram-positive and fungal strains.

\section{MATERIALS AND METHODS}

\subsection{MATERIALS}

Cashew gum (CG) were kindly donated by the Laboratory of Polymers of the Federal University of Ceará (Fortaleza, Brazil). Crude samples were extracted from native Anacardium accidentale L. tree, isolated and purified according methodology previously described by Paula and co-workers[15]. All other reagents were of analytical grade (Formamide, pyridine, acetic anhydride, dimethyl sulfoxide were purchase from Vetec and pyrene was obtained from Fluka).

\subsection{METHODS}

\subsubsection{SYNTHESIS OF ACETYLATED CASHEW GUM (ACG)}

CG polysaccharide was chemically modified with acetyl moieties by the methodology proposed by Mozato and collaborators as reported in Pitombeira et al. [7] This step is to induce amphiphilic properties in the material. Briefly, CG $(1 \mathrm{~g})$ was suspended in $20 \mathrm{~mL}$ of formamide and dissolved with vigorous stirring at $50^{\circ} \mathrm{C}$. Pyridine $(6 \mathrm{~mL})$ and acetic anhydride $(15 \mathrm{~mL})$ were added in the bulk and the mixture was stirred for $24 \mathrm{~h}$. Acetylated cashew gum (ACG) was precipitated using $400 \mathrm{~mL}$ of water, with further filtration and washing with double-deionized water. The recovered material was dried in hot air[7].

The modification of CG was characterized by Infrared Spectroscopy (FT-IR Shimadzu 8300 spectrophotometer) and magnetic resonance spectroscopy using a Fourier transform Bruker Avance DRX 500 spectrometer with an inverse multinuclear gradient probe-head equipped with z-shielded gradient coils and a Silicon Graphics workstation (data not shown). FT-IR analysis were recorded obtaining $\mathrm{KBr}$ pellets with the samples, in the range of 4,000 to $400 \mathrm{~cm}^{-1}$, with a resolution of $2 \mathrm{~cm}^{-1}$ and 15 scans. ${ }^{1} \mathrm{H}$ NMR $(400 \mathrm{MHz}$, DMSO-d6) spectra of $3 \% \mathrm{w} / \mathrm{v}$ were recorded at $353 \mathrm{~K}$. Sodium 2,2-dimethylsilapentane-5- sulphonate (DSS) was used as the internal standard [10].

\subsubsection{Synthesis ACG nanoparticles}

ACG nanoparticles were prepared according to the method described elsewhere [7,10]. Briefly, ACG (20 $\mathrm{mg}$ ) was dissolved in $20 \mathrm{~mL}$ of dimethyl sulfoxide (DMSO) and the solution was submitted to agitation with magnetic stirring during 30 minutes to ensure complete homogenization, with subsequent dialysis for $24 \mathrm{~h}$ (molecular weight cut-off14 kD) in double-deionized water $(600 \mathrm{~mL})$. Water conductivity was the check point to monitor the water changing intervals, which should be similar to double-deionized water (approximately after 3 days). After dialysis, the nanoparticles were collected from the interior of the membrane, and suspended in known volume of ultrapure water, and further freeze-dried when appropriate. 


\subsection{CHARACTERIZATION OF CASHEW GUM NANOPARTICLES}

\subsubsection{Particle size, polydispersity index and zeta potential}

Particle size and zeta potential measurements were carried out on a Malvern Zetasizer Nano, Model ZS 3600, analyzer. The hydro-dynamic diameter of the obtained ACG nanoparticles was measured by dynamic light scattering (DLS) with laser at a wavelength of $633 \mathrm{~nm}$ and a fixed scattering angle of $173^{\circ}$. Each sample was measured three times, under room temperature.

\subsubsection{Determination of nanoparticles concentration by particle tracking analysis}

Nanoparticle tracking analysis (NTA) combines laser light scattering microscopy with a charge-coupled device (CCD) camera, which enables the visualization and recording of nanoparticles in solution. The NTA software is then able to identify and track individual nanoparticles moving under Brownian motion and relates the movement to a particle size according to the following formula derived from the Stokes-Einstein Equation 1:

$$
\overline{(x, y)^{2}}=\frac{2 K_{b} T}{3 r h \pi \eta}
$$

where $\mathrm{K}_{\mathrm{b}}$ is the Boltzmann constant and $(\mathrm{x}, \mathrm{y})^{2}$ is the mean-squared speed of a particle at a temperature $\mathrm{T}$, in a medium of viscosity $\eta$, with a hydrodynamic radius of rh.

The colloidal stability of the obtained ACG nanoparticles was assessed by storing the suspensions in preset temperature and humidity conditions during five months. The stability of the nanoparticles was evaluated comparing the zeta potential of the particles before and after storage.

\subsubsection{Colloidal stability studies}

The colloidal stability of the obtained ACG nanoparticles was assessed by storing the suspensions in preset temperature and humidity conditions during five months. The stability of the nanoparticles was evaluated comparing the zeta potential of the particles before and after storage.

\subsection{ANTIBACTERIAL AND ANTIFUNGAL ACTIVITY}

\subsubsection{Selected strains and growth conditions}

For the evaluation of antibacterial activity of the nanoparticles, the assays sere performed with S. aureus (ATCC 25923) and E. coli (ATCC 25922) strains. For the evaluation of antifungal activity, C. albicans (NEWP31). Muller-Hinton broth and MH agar (Difco, USA) were used as growth media. The strains were maintained in inclined agar, under temperature of $4^{\circ} \mathrm{C}$. Cultures were obtained in Brain Hearth Infusion BHI (Difco Laboratoires) broth before the assays.

\subsubsection{Direct contact assay}

For this assay, suspension containing ca. $1.5 \times 10^{8} \mathrm{CFU}$ (colony forming unit) $\mathrm{mL}^{-1}$ were prepared in saline solutions, following the MacFarland scale [16]. Further, $100 \mu \mathrm{L}$ of this suspension was transferred to petri dishes containing agar Mueller Hinton. In each dish, $100 \mu \mathrm{L}$ of the nanosuspensions were seeded with a Drigalsky's handle by the spread plate method, and incubated at $37^{\circ} \mathrm{C}$, for $24 \mathrm{~h}$. Dishes with saline solution instead nanoparticles suspensions were used as control. DSMO was used as solvent control. The inhibitory effect of each sample was calculated according the following equation:

$$
\eta=\frac{N_{1}-N_{2}}{N_{1}}
$$

where $\eta$ is inhibitory effect, $N_{1}$ is the mean of UFC of the control dishes, and $\mathrm{N}_{2}$ is the mean of UFC of the tested samples. 


\subsection{STATISTICAL ANALYSIS}

All the data were analyzed by the Turkey test ANOVA, considered statistically significant when $p$ value < 0.05 . The results were expressed as mean and \pm standard deviation.

\section{RESULTS AND DISCUSSION}

\subsection{Characterization of a CG nanoparticles}

\subsubsection{Particle size, polydispersity index and zeta potential}

For the development of ACG nanoparticles, the nanoprecipitation by dialysis method was selected. The formation of the nanoparticles is based on the slow exchange of the organic solvent with the non-solvent water during dialysis, allowing the precipitation of the polymers into nano-sized scale[17].This method is advantageous due to its low cost, easy application and absence of emulsifiers, in addition to be useful when solvent removal is not possible by evaporation [17].

Previously, Pitombeira and co-worker developed nanoparticles using ACG for drug delivery purposes using DMSO as solvent founding, good parameters regarding particles size, PDI and zeta potential[7]. The nature of the solvent chosen to obtain polysaccharide nanoparticles directly influences on the size and PDI. These parameters were mainly found to be dependent on interfacial tension and viscosity of the solvent, as already studied before[18].

Acetone is one of the most preferred solvents to produce nanoparticles by dialysis method as its rapid diffusion from the emulsion droplets from the polymer solution to water promotes an interfacial turbulence which contributes for the spontaneous formation of nano-sized particles[19]. Moreover, it is less toxic and easy to be removed due to low boiling point, avoiding solvent residual in the final colloidal suspension[17]. In this work, DMSO (C1) and acetone (C2) were used to produce ACG nanoparticles. The obtained data are expressed in Table 1.

Table 1: Characterization of the ACG nanoparticles regarding size, polydispersity index (PDI) and zeta potential by dynamic light scattering.

\begin{tabular}{ccccc}
\hline Solvent & Sample & Particle Size & PDI & Zeta potential \\
\hline DMSO & C1 & $81.3 \pm 0.1$ & $0.219 \pm 0.009$ & -39.9 \\
\hline Acetone & C2 & $115.7 \pm 1.3$ & $0.122 \pm 0.015$ & -31.7
\end{tabular}

Based on the obtained data, it was observed that the particles were statistically different $(p<0.05)$ regarding particle size and PDI, using different solvents. Lower nanoparticles were observed when using DMSO as solvent $(81.3 \mathrm{~nm})$, compared to those obtained with acetone (115.7) (Table 1). The histograms of the obtained nanoparticles are present in Figure 1.

Both $\mathrm{C} 1$ and $\mathrm{C} 2$ samples showed narrow distribution, presenting PDI values of $0.219 \pm 0.009$ and 0.122 \pm 0.015 respectively. Although, acetone originated particles with more homogeneous distribution (lower PDI value). These results are in accordance with results previously reported ACG to produce nanoparticles by dialysis method $[7,10]$. Same findings were observed by Jeong et al., who studied the influence of different solvent, including DMSO and acetone, on the particle size and PDI, in which nanoparticles produced with DMSO showed to be smaller than those produced with acetone[20]. This change in particle size could be explained by the difference in solvent viscosity, miscibility with water and in the solubility behavior of ACG[21].

The different sizes of nanoparticles formed are assigned to the different chemical properties of organic solvents. The average sizes of nanoparticles increase or decrease according to the solubility parameters of the organic solvents. 

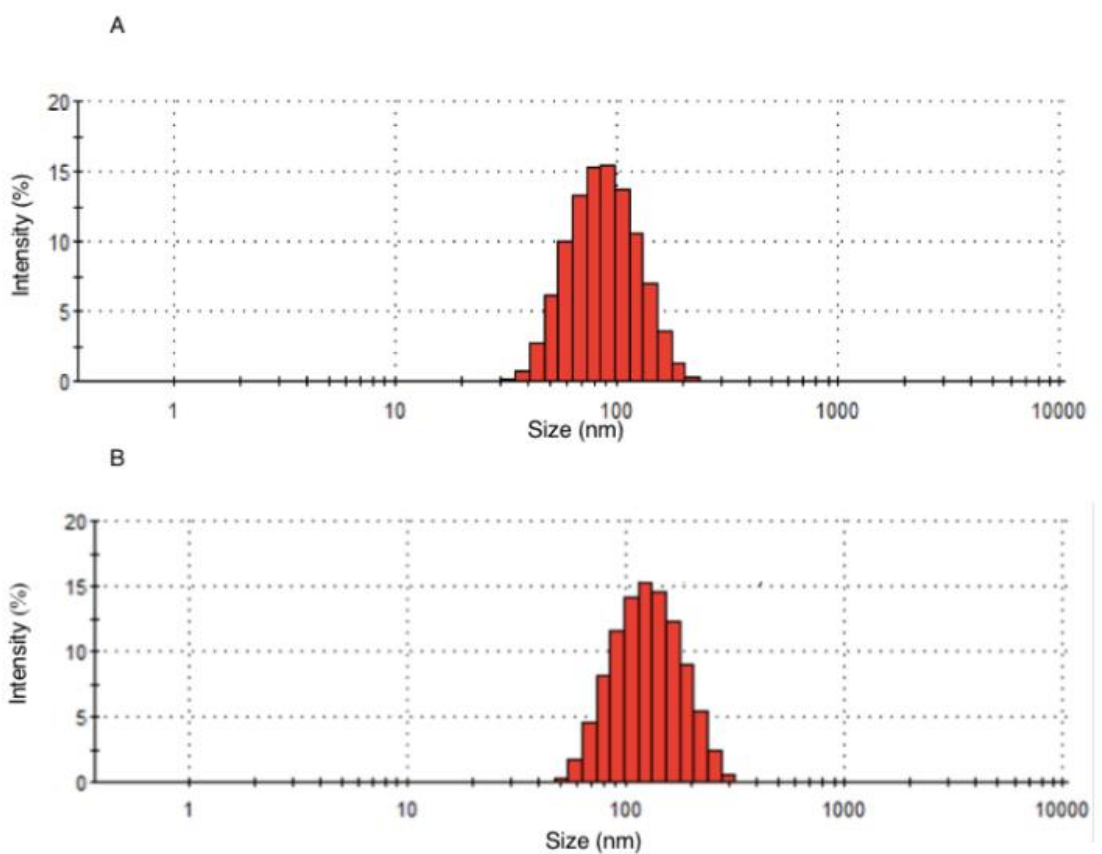

Figure 1: Histogram distribution of ACG nanoparticles prepared with DMSO (A) and Acetone (B).

The boiling point of solvent is related to evaporation capacity. Higher boiling point, lower its evaporation rate or volatility. The dipole moment is directly proportional related to the polarity of solvent, that is, greater dipole moment and greater polarity. Comparing the values of the described properties (Table 2), DMSO presents higher boiling point and dipole moment. It can suggest the greater difficulty of this solvent in evaporate of the system together with its greater polarity results in greater difficulty of the gum to interact with itself to form NP in relation to acetone. Therefore, it is expected that NPs generated in DMSO are lower when compared to those formed in acetone.

Table 2: Physicochemical parameters of DMSO and acetone.

\begin{tabular}{|c|c|c|c|c|c|c|c|}
\hline \multirow[b]{2}{*}{ Solvent } & \multirow[b]{2}{*}{$\begin{array}{l}\text { Boiling Point } \\
\qquad\left({ }^{\circ} \mathrm{C}\right)\end{array}$} & \multirow[b]{2}{*}{ Dipole Moment (D) } & \multirow[b]{2}{*}{$\begin{array}{c}\text { Dieletric } \\
\text { Constant }(\varepsilon)\end{array}$} & \multicolumn{4}{|c|}{$\begin{array}{l}\text { Hansen parameters } \\
\qquad\left(\mathrm{Mpa}^{\mathbf{1 / 2}}\right)\end{array}$} \\
\hline & & & & $\delta \mathrm{t}$ & $\delta \mathrm{d}$ & $\delta \mathbf{p}$ & $\delta \mathbf{h}$ \\
\hline Acetone & 56.1 & 2.69 & 20.56 & 20 & 15.5 & 10.4 & 7 \\
\hline DMSO & 189.1 & 4.06 & 46.45 & 26.7 & 18.4 & 16.4 & 10.2 \\
\hline
\end{tabular}

The measurements of zeta potential revealed that both $\mathrm{C} 1$ and $\mathrm{C} 2$ present negative charge on the surface of the nanoparticles, and values higher than $30 \mathrm{mV}$ in modulus, suggesting that the colloidal system is stable due to the repulsion of the particles.

\subsubsection{Determination of nanoparticles concentration by particles tracking analysis}

Particle tracking analysis (PTA) has been commonly used to measure physical parameters of nanoparticles as well as to correlate the number of particles analyzed to concentration. The main advantage of PTA over DLS is that this technique can detect small, weakly-scattering particles among large, strong scatterers that would dominate the size distribution determined by DLS, avoiding false results[22].

According to the results, the concentrations of nanoparticles founded for $\mathrm{C} 1$ and $\mathrm{C} 2$ were respectively $9.24 \pm 0.03 \times 10^{10}$ and $1.87 \pm 0.07 \times 10^{10}$ particles $\mathrm{mL}^{-1}$ (Figure 2 ). It could be seen that a slight increase in 
the concentration of the nanoparticles were found when the solvent used was DMSO, which corroborate with the particle size analysis in which $\mathrm{C} 1$ presented lower size compared to $\mathrm{C} 2$.

In the case of this study, nanoparticles were obtained by promoting the supersaturation of the ACG in the selected solvents. It well known that each substance has different degree of saturation depending on their properties and the solvent characteristic, including degree of polarity. Moreover, interactions between polymer-solvent-water greatly influences the precipitation process as each solvent enable polymer moieties to diffuse at different rates in the ternary system, which affect growth at the solid-liquid interface[22].

According to the obtained results, it was possible to infer that ACG is more soluble in DMSO than in acetone, thus the organic solution would be more concentrated, resulting in more concentrated colloidal suspension. In addition, DMSO-ACG mixture may present a lower diffusivity index compared to acetone-ACG, leading to the obtainment of lower particle size since lower diffusion rates slows particle formation[22].
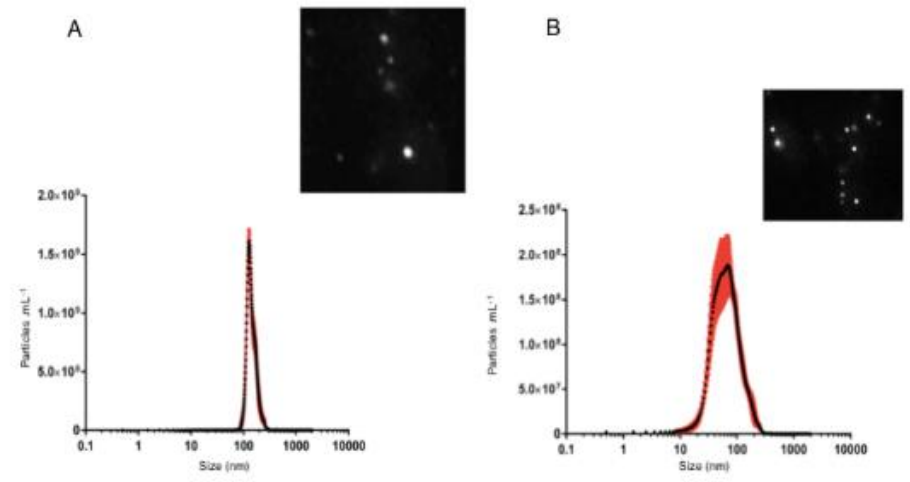

Figure 2: Particle tracking analysis of $\mathrm{C} 1(\mathrm{~A})$ and $\mathrm{C} 2(\mathrm{~B})$.

\subsubsection{Colloidal stability studies}

The colloidal stability of the ACG nanoparticles obtained with DMSO (C1) and acetone (C2) was evaluated regarding zeta potential as this parameter measures the magnitude of the electrostatic interactions, which is crucial for the stability of colloidal systems[23]. In addition, possible changes in zeta potential over storage could be attributed to solvent residual, thus it was important to evaluate this parameter. Colloidal stability of both $\mathrm{C} 1$ and C2 ACG nanoparticles was evaluated before and after 5 months, and no statistical differences (p $<0.05$ ) between the same samples was found after this period (Figure 3), confirming that both DMSO and acetone are suitable to produce stable nanoparticles.

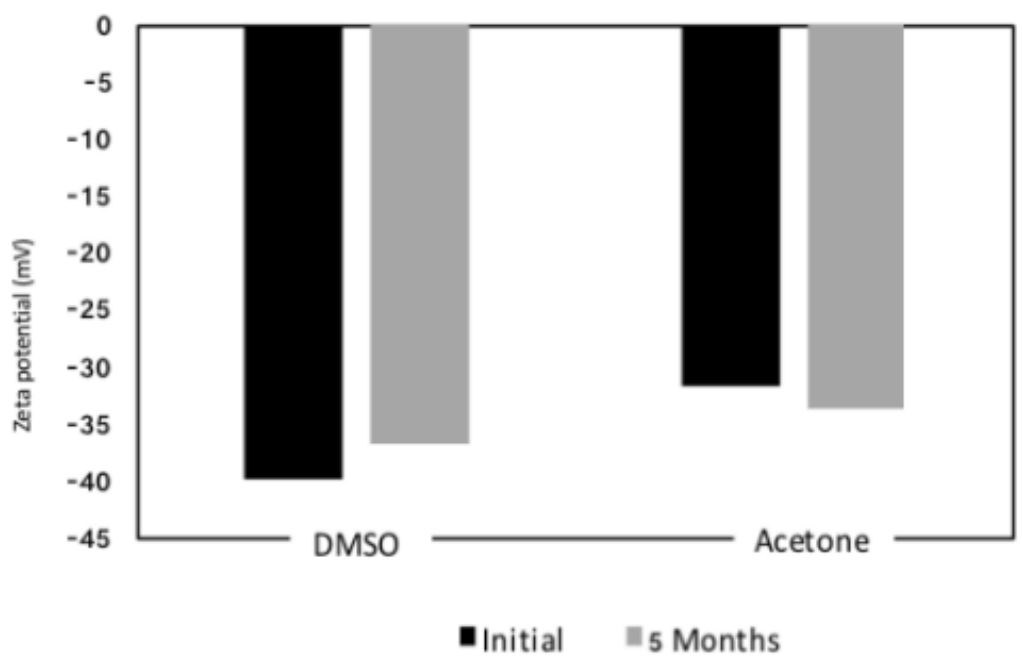

Figure 3: Zeta potential of DMSO (C1) and acetone (C2) ACG nanoparticles before and after 5 months. 


\subsection{Antibacterial and antifungal activity}

The antibacterial and antifungal activity was performed with Gram-negative (E. coli), Gram-positive ( $S$. aureus) and fungi (C. albicans) strains aiming to evaluate significant antimicrobial activity among species with different characteristic, under contact during $24 \mathrm{~h}$. C. albicans was chosen to evaluate antifungal activity as it is a human pathogen commonly found, in addition to be recurrent in hospitalized patients. The obtained results are express in Table 3.

The experiments realized with E. coli (EC) with the tested samples did not present significant difference compared to positive control on the contrary of the experiments realized with $S$. aureus. These results reveal that the chemical composition of bacterial membrane is determinant to induce resistance or susceptibility to entry of substances inside bacteria. The activity against SA may be explained by the facilitated penetration of the nanoparticles over the bacteria cell wall, which is composed by a thick peptidoglycan layer with hydrophobic characteristic, on the contrary of EC cell wall, in which the peptidoglycan layer thinner, having no affinity with the nanoparticles.

Regarding antifungal activity, it was possible to observe that samples obtained with DMSO (C1) presented a more pronounced antifungal effect $(81.2 \%)$, compared to nanoparticles produced with acetone (C2) (18.8\%). This effect could be explained by the difference of nanoparticles concentration between $\mathrm{C} 1$ (92.4 x $10^{9}$ particles $\mathrm{mL}^{-1)}$ and $\mathrm{C} 2\left(18.8 \times 10^{9}\right.$ particles $\left.\mathrm{mL}^{-1}\right)$, in addition to the smaller size of $\mathrm{C} 1$ nanoparticles. The cellular membrane of fungi is composed by glycans, soluble and insoluble polysaccharides and chitin, presenting a hydrophobic characteristic, which seems to facilitate the passage of insoluble compounds through the membrane, similarly to gram-positive bacteria.

Table 3: Inhibitory effect of tested samples after 24h of contact with S. aureus (SA) and C. albicans (CA).

\begin{tabular}{|c|c|c|c|c|}
\hline \multirow[t]{2}{*}{ Sample } & \multirow{2}{*}{$\begin{array}{c}\text { Concentration } \\
\left(10^{9} \cdot \mathrm{mL}^{-1}\right)\end{array}$} & \multirow[t]{2}{*}{ Size $(n m)$} & \multicolumn{2}{|c|}{ Inhibitory effect } \\
\hline & & & C. albicans (CA) & S. aureus (SA) \\
\hline C1 & 92.4 & 81.3 & 81.2 & 36.9 \\
\hline C2 & 18.8 & 115.7 & 18.7 & 19.9 \\
\hline
\end{tabular}

\section{CONCLUSIONS}

In this work, ACG based self-assembled nanoparticles were successfully produced by dialysis method, using different solvent, namely DMSO and acetone, evidencing that there were significant differences regarding particle size and PDI values. On the other hand, unimodal particle size and homogeneous distributions were observed using dynamic light scattering for particles produced with both DMSO and acetone. Moreover, both $\mathrm{C} 1$ and $\mathrm{C} 2 \mathrm{ACG}$ nanoparticles showed good colloidal stability over 5 months confirming that both DMSO and acetone are suitable to produce stable nanoparticles. PTA evidenced that the using DMSO as solvent a more concentrated suspension was produced, which corroborate with the particle size analysis. Both $\mathrm{C} 1$ and C2 showed to be stable regarding zeta potential after 5 month of storage, confirming that both DMSO and acetone are suitable to produce stable nanoparticles. Regarding antimicrobial activity, none of tested sample was effective against E. coli strain, on the contrary of S. aureus, in which both C1 (36.9\%) and C2 (19.9\%) resented significant inhibitory effect. These results suggest that ACG nanoparticles have more affinity to gram-negative bacteria, probably due to the presence of a more complex cell wall, with more hydrophobic characteristic. Higher inhibitory effects were found against $C$. albicans, specially with particles produced with DMSO (81.2\%), probably due to its higher concentration of nanoparticles. Overall, both nanoparticles presented suitable physicochemical characteristics, and showed an important antimicrobial effect against gram-negative strain (S. aureus) and fungal (C. albincas), indicating a promising platform to be used in several biomedical application.

\section{ACKNOWLEDGEMENTS}

The authors thank the Conselho Nacional de Desenvolvimento Científico e Tecnológico - CNPq, the Coordenação de Aperfeiçoamento de Pessoal de Nível Superior - CAPES and the Fundação de Amparo à Pesquisa do Estado do Piauí. All authors contributed equally to this work. 


\section{BIBLIOGRAPHY}

[1] DE OLIVEIRA, E.F., PAULA, H.C.B., PAULA, R.C.M. “Alginate/cashew gum nanoparticles foressential oil encapsulation”, Colloids Surfaces B Biointerfaces, v. 113, pp. 146-151, 2014.

[2] FRIEDMAN, A.J., PHAN, J., SCHAIRER, D.O., et al. "Antimicrobial and anti-inflammatory activity of chitosan-alginate nanoparticles: A targeted therapy for cutaneous pathogens," J. Invest. Dermatol., v. 133, n. 5, pp. 1231-1239, 2013.

[3] SALEM, W., LEITNER, D.R., ZINGL, F.G., et al. "Antibacterial activity of silver and zinc nanoparticles against Vibrio cholerae and enterotoxic Escherichia coli," Int. J. Med. Microbiol., v. 305, n. 1, pp. 85-95, 2015.

[4] DI MARTINO, A., SEDLARIK, V., “Amphiphilic chitosan-grafted-functionalized polylactic acid based nanoparticles as a delivery system for doxorubicin and temozolomide co-therapy," Int. J. Pharm., v. 474, n. 1-2, pp. 134-145, 2014.

[5] RIBEIRO, A.J., DE SOUZA, F.R.L., BEZERRA, J.M.N.A., et al.“Gums' based delivery systems: Review on cashew gum and its derivatives,” Carbohydr. Polym., v. 147, pp. 188-200, 2016.

[6] YANG, J., HAN, S., ZHENG, H., et al., "Preparation and application of micro/nanoparticles based on natural polysaccharides," Carbohydr. Polym., v. 123, pp. 53-66, 2015.

[7] PITOMBEIRA, N.A.O., VERAS NETO, J.G., SILVA, D.A., et al., "Self-assembled nanoparticles of acetylated cashew gum: Characterization and evaluation as potential drug carrier," Carbohydr. Polym., v. 117, pp. 610-615, 2015.

[8] QUELEMES, P.V., ARARUNA, F.B., DE FARIA, B.E.F., et al., "Development and antibacterial activity of cashew gum-based silver nanoparticles,” Int. J. Mol. Sci., v. 14, n. 3, pp. 4969-4981, 2013.

[9] CLARA M.W.S., ABREUA, H. C.B., PAULA, B., et al."Synthesis and characterization of non-toxic and thermo-sensitive poly(N-isopropylacrylamide)-grafted cashew gum nanoparticles as a potential epirubicin delivery matrix," Front. Behav. Neurosci., v. 6, pp. 77-85, 2016.

[10] DIAS, S. F. L., NOGUEIRA, S. S., FRANÇA DOURADO, F., et al., "Acetylated cashew gum-based nanoparticles for transdermal delivery of diclofenac diethyl amine," Carbohydr. Polym., v. 143, pp. 254-261, 2016.

[11] CAMPOS, D. A., RIBEIRO, A. C., COSTA, E. M.; et al. "Study of antimicrobial activity and atomic force microscopy imaging of the action mechanism of cashew tree gum," Carbohydr. Polym., v. 90, n. 1, pp. 270-274, 2012.

[12] ARAÚJO, I. M. S., ZAMPA, M. F., MOURA, J. B., et al.“Contribution of the cashew gum (Anacardium occidentale L.) for development of layer-by-layer films with potential application in nanobiomedical devices,” Mater. Sci. Eng. C, v. 32, n. 6, pp. 1588-1593, 2012.

[13] KUBO, I., MUROI, H., KUBO, A., "Structural functions of antimicrobial long-chain alcohols and phenols," Bioorganic Med. Chem., v. 3, n. 7, pp. 873-880, 1995.

[14] LIMA, M. R., PAULA, H. C. B., ABREU, F. O. M. S., et al. "Hydrophobization of cashew gum by acetylation mechanism and amphotericin B encapsulation," Int. J. Biol. Macromol., v. 108, pp. 523-530, 2018.

[15] DE PAULA, R.C., HEATLEY, F.B.P. "Characterization of Anacardium occidentale exudate polysaccharide,” v. 45, n. 1, p. 1998, 1998.

[16] MCFARLAND, J. "The Nephelometer:an Instrument for Estimating the Number of Bacteria in Suspensions Used for Calculating the Opsonic Index and for Vaccines.," The Journal of the American Medical Association., vol. XLIX, n. 14, p. 1176, 1907.

[17] ALMOUSTAFA, H. A., ALSHAWSH, M. A., CHIK, Z. "Technical aspects of preparing PEG-PLGA nanoparticles as carrier for chemotherapeutic agents by nanoprecipitation method", Int. J. Pharm., v. 533, n. 1, pp. 275-284, 2017.

[18] GAVORY, C., DURAND, A., SIX, J. L., et al., "Polysaccharide-covered nanoparticles prepared by nanoprecipitation," Carbohydr. Polym., v. 84, n. 1, pp. 133-140, 2011.

[19] SAH, E., SAH, H., "Recent trends in preparation of poly(lactide-co-glycolide) nanoparticles by mixing polymeric organic solution with antisolvent," J. Nanomater., v. 2015, 2015.

[20] JEONG, Y.I., CHO, C.S., KIM, S.H., et al., "Preparation of poly (DL-lactide- co-glycolide) nanoparticles without surfactant.," J. Appl. Polym. Sci., v. 80, n. 12, p. 11, 2001. 
[21] CRUCHO, C. I. C., BARROS, M. T. "Polymeric nanoparticles: A study on the preparation variables and characterization methods," Mater. Sci. Eng. C, v. 80, pp. 771-784, 2017.

[22] GALLEGO-URREA, J. A., TUORINIEMI, J., HASSELLÖV, M. “Applications of particle-tracking analysis to the determination of size distributions and concentrations of nanoparticles in environmental, biological and food samples," TrAC - Trends Anal. Chem., v. 30, n. 3, pp. 473-483, 2011.

[23] MARTÍNEZ RIVAS, C. J., TARHINI, M., BADRI, W., et al., "Nanoprecipitation process: From encapsulation to drug delivery," Int. J. Pharm., v. 532, n. 1, pp. 66-81, 2017.

\section{ORCID}

Karolina Carneiro Morais Macedo Luíse Lopes Chaves

Alexandre Couto Carneiro Vieira

Daniela Nadvorny

Regina Célia Monteiro de Paula

Durcilene Alves Silva

Francisco Veiga

António José Ribeiro

Edson Cavalcanti Silva-Filho http://orcid.org/0000-0001-7235-3401

http://orcid.org/0000-0002-1197-7306

http://orcid.org/0000-0002-9399-8169

http://orcid.org/0000-0001-9154-764X

http://orcid.org/0000-0002-7617-0343

http://orcid.org/0000-0001-8698-7309

http://orcid.org/0000-0002-1041-0068

http://orcid.org/0000-0002-1399-8944

http://orcid.org/0000-0003-0988-2970 